\title{
Suporte ético-emocional à profissionais de enfermagem frente à pandemia de COVID-19: relato de experiência
}

\author{
Ethical-emotional support for Nursing professionals facing the COVID-19 pandemic: An experience report \\ Soporte ético-emocional a profesionales de enfermería ante la pandemia COVID-19: relato de \\ experiencia
}

\section{Gabriela Gonçalves Amaral ${ }^{1,2}$ [C] Lívia Silveira Silva ${ }^{2,3}$ (1) Jarbas Vieira de Oliveira ${ }^{2,4}$ (D) Noelle Melo Machado ${ }^{2}$ (1) Juliana Silveira Teixeira 2,5 (1) Hozana Reis Passos $2,4,6$ [C]}

1. Universidade de São Paulo. Ribeirão Preto, SP, Brasil.

2. Conselho Regional de Enfermagem de Minas Gerais. Belo Horizonte, MG, Brasil. 3. Universidade Federal de São João del-Rei. Divinópolis, MG, Brasil.

\section{Prefeitura de Belo Horizonte. Belo} Horizonte, MG, Brasil.

\section{Santa Casa de Misericórdia de Belo} Horizonte. Belo Horizonte, MG, Brasil.

6. Universidade Federal de Minas Gerais. Belo Horizonte, MG, Brasil.

Autor correspondente:

Hozana Reis Passos.

E-mail: hozanapassos@gmail.com

Recebido em 07/06/2021.

Aprovado em 07/09/2021.

DOl:https://doi.org/10.1590/2177-9465-EAN-2021-0234

\begin{abstract}
Resumo
Objetivo: Descrever a experiência de planejamento, execução e avaliação de um serviço de suporte ético-emocional para profissionais de enfermagem frente à pandemia de COVID-19. Método: Relato de experiência no âmbito da Comissão de Suporte Ético-Emocional do Conselho Regional de Minas Gerais, Brasil, desenvolvido entre os meses de abril e dezembro de 2020 e operacionalizado através de ligações telefônicas e mensagens via aplicativo de comunicação. Resultados: Foram atendidos 241 profissionais. Foram revelados e declarados sentimentos, emoções, vivências e problemas oriundos do cotidiano de trabalho da enfermagem diante do contexto pandêmico. Tais problemas reforçam a eminência, a urgência e o valor que 0 suporte teve para a vida, trabalho e saúde dos profissionais atendidos, configurando-se como uma estratégia de saúde e de intervenção indispensável à promoção, prevenção, segurança e proteção da saúde mental frente à pandemia. Os atendimentos culminaram na redução de sentimentos negativos; na percepção aumentada (identificação de potenciais ameaças e contextos de "gatilhos"); na valorização pessoal; no autoconhecimento e autocuidado. Considerações finais e implicações para a prática: O suporte fez-se inovador para área da saúde e da enfermagem ao constituir-se como uma estratégia multidisciplinar promotora, aconselhadora e facilitadora do cuidado em tempos de COVID-19. Encoraja-se a manutenção desta estratégia após a pandemia.
\end{abstract}

Palavras-chave: COVID-19; Enfermagem; Profissionais de Enfermagem; Saúde Mental; Telemedicina.

\section{Abstract}

Objective: To describe the experience of planning, implementing and evaluating an ethical-emotional support service for Nursing professionals facing the COVID-19 pandemic. Method: An experience report in the scope of the Ethical-Emotional Support Commission of the Regional Council of Minas Gerais, Brazil, developed between April and December 2020 and operationalized through phone calls and messages via a communication application. Results: A total of 241 professionals were assisted. Feelings, emotions, experiences and problems arising from the daily Nursing work in the pandemic context were revealed and stated. Such problems reinforce the prominence, urgency and value that the support service had for the life, work and health of the professionals assisted, configuring itself as a health and intervention strategy, indispensable for mental health promotion, prevention, safety and protection in the face of the pandemic. The consultations culminated in the reduction of negative feelings; in increased perception (identification of potential threats and "trigger" contexts); in personal appreciation; and in self-knowledge and self-care. Final considerations and implications for the practice: The support service was innovative for the Health and Nursing areas by constituting a multidisciplinary strategy that promotes, counsels, and facilitates care in times of COVID-19. We encourage the maintenance of this strategy after the pandemic.

Keywords: COVID-19; Mental Health; Nursing; Nursing Professionals; Telemedicine.

\section{RESUMEN}

Objetivo: Describir la experiencia de planificación, implementación y evaluación de un servicio de soporte ético-emocional para los profesionales de enfermería ante la pandemia de COVID-19. Método: Relato de experiencia en el ámbito de la Comisión de Soporte Ético-Emocional del Consejo Regional de Minas Gerais, Brasil, desarrollado entre los meses de abril y diciembre de 2020 y operacionalizado a través de enlaces telefónicos y mensajes vía la aplicación de comunicación. Resultados: Se atendió a 241 profesionales. Se revelaron y declararon los sentimientos, las emociones, las experiencias y los problemas derivados del trabajo diario de las enfermeras ante el contexto de la pandemia. Estos problemas refuerzan la eminencia, la urgencia y el valor que e soporte tiene para la vida, el trabajo y la salud de los profesionales atendidos, configurándose como una estrategia de salud y de intervención indispensable para la promoción, prevención, seguridad y protección de la salud mental frente a la pandemia. Las consultas culminaron en la reducción de los sentimientos negativos; en el aumento de la percepción (identificación de posibles amenazas y contextos "desencadenantes"); en la valoración personal; en el autoconocimiento y el autocuidado. Consideraciones finales e implicaciones para la práctica: El soporte fue innovador en el ámbito de la salud y la enfermería como estrategia multidisciplinaria para promover, asesorar y facilitar la asistencia en tiempos de COVID-19. Se fomenta el mantenimiento de esta estrategia después de la pandemia.

Palabras claves: COVID-19; Enfermería; Profesionales de Enfermería; Salud Mental; Telemedicina. 


\section{INTRODUÇÃO}

O bem-estar, a segurança e a proteção dos profissionais de saúde, devem representar e esculpir o escopo primaz de ações, incentivos e políticas de saúde ${ }^{1}$. Assim, independente do contexto social-global, salvaguardar os profissionais de saúde, de forma a lhes oferecer orientações, apoio e suporte, configura-se como uma das medidas essenciais à manutenção, promoção e proteção da saúde física e mental, especialmente no contexto da pandemia de COVID-19².

Dessa forma, frente à situação pandêmica, o Conselho Regional de Enfermagem de Minas Gerais (COREn-MG), órgão de regulamentação do exercício profissional da enfermagem, instituiu a Comissão de Suporte Ético-Emocional (CSEE) articulada ao Comitê Gestor de Crise do respectivo conselho, por meio da portaria $\mathrm{n}^{\circ} \mathbf{1 6 6}$, de 30 de março de $2020^{3}$. A CSEE visava sobretudo, acolher essas pessoas diante das repercussões da COVID-19 frente a vida, o trabalho e a saúde dos profissionais de enfermagem. A ideia dessa intervenção, implicou em ações objetivas e concretas, pautadas na realidade dos profissionais mineiros, que neste caso, foi prioritariamente o atendimento ao sofrimento ético-emocional ${ }^{3}$.

Neste contexto, destaca-se a importância da prestação de suporte ético-emocional na manutenção, prevenção, promoção e proteção dos trabalhadores de Enfermagem durante o cenário pandêmico. Notabiliza-se também, a relevância desse suporte ao ser conduzido por enfermeiras (os) especialistas em saúde mental e/ou no atendimento individualizado para toda a categoria de enfermagem. Afinal, além da oferta do cuidado contínuo e especializado, a Enfermagem trabalha visando a ação terapêutica construída a partir das subjetividades e complexidades humanas. Na COVID-19, a Enfermagem escuta e acolhe com sensibilidade e cientificidade, principalmente à dimensão emocional do ser humano ${ }^{4}$.

A partir desse cenário, surgiu o seguinte questionamento: quem cuidará de quem cuida? Logo, este relato objetiva-se descrever a experiência de planejamento, execução e avaliação de um serviço de suporte ético-emocional para profissionais de enfermagem frente à pandemia de COVID-19. O texto sumariza algumas intervenções e estratégias que favoreceram e sensibilizaram a promoção e proteção da saúde mental dos profissionais de enfermagem, proporcionando-lhes uma atenção segura e qualificada, ao envolver o suporte e o apoio éticoemocional, bem como demandas apontadas e a satisfação dos mesmos com o suporte.

\section{DESCRIÇÃO DA EXPERIÊNCIA}

Os relatos de experiência narram e descrevem a experiência vivida articulando-as ao cotidiano científico-teórico-prático ${ }^{5}$. Assim, este relato deriva-se de uma experiência no âmbito da CSEE do COREn-MG, sediado em Belo Horizonte, Minas Gerais, Brasil, desenvolvido entre os meses de abril e dezembro de 2020. Dada a situação de isolamento e distanciamento social necessários à prevenção de contágio da COVID-19, este suporte foi operacionalizado através de ligações telefônicas e mensagens via aplicativo de comunicação (com número oficial e destinado a esse fim) e ainda contou com uma equipe de enfermeiras (os) qualificadas (os) na assistência em saúde mental e capacitados de forma a executar a organização do serviço e dos processos de atendimento.

O suporte iniciou-se a partir do agendamento prévio, pelos inscritos, via canais de comunicação do COREn-MG (página eletrônica e número institucional de um aplicativo de comunicação). A oferta de atendimentos foi definida a partir de alguns critérios: 1) ser profissional de enfermagem (enfermeiro, técnico, auxiliare obstetrizes); 2) estar inscrito no COREn-MG; 3) podendo estar: de férias, afastado do trabalho, desempregado ou atuando no setor público ou privado durante a pandemia de COVID-19; e 4) dispor de um número telefônico atualizado. Assim, enquadrandose nesses critérios, o profissional de enfermagem (identificado pela CSEE como “inscrito") podia buscar o agendamento para o suporte. Para o agendamento, era necessário informar:a) nome completo ou pseudônimo; b) categoria profissional; c) número de telefone para contato; d) dia e hora preferenciais para o atendimento. Aos inscritos que optaram pelo agendamento via aplicativo de comunicação, Ihes era dada a opção do suporte via ligação telefônica ou através do chat de mensagens do próprio aplicativo, em número de contato dedicado ao agendamento do suporte. Ressalta-se que, em todo seu desenvolvimento, a CSSE adotou e considerou os preceitos éticos que envolvem os seres humanos. Dessa forma, informações como o nome completo, a categoria profissional e o número de telefone eram mantidos somente para controle e matriciamento da própria comissão. Ademais, o inscrito que optava pelo agendamento via aplicativo de comunicação recebia imediatamente uma mensagem de confirmação do agendamento com o dia, hora e nome completo do (a) profissional enfermeiro (a) que iria atendê-lo. Para opção de agendamento via site, essa mensagem era enviada 24 horas antes do dia previsto para o atendimento.

Vale ressaltar que a CSSE tinha horários disponíveis de segunda a sexta-feira das 8 às 21 horas, nesses horários haviam pelo menos dois profissionais disponíveis para atendimento. Por se tratar de um serviço online e de cunho emocional ao longo do trabalho desenvolveu-se critérios para uma classificação de risco à distância que se baseava: a) no teor da conversa do inscrito; b) na sua performance vocal (voz trêmula, chorosa, ofegante etc), observados pela ligação e ou áudios do aplicativo de comunicação; c) na imagem do perfil do inscrito no aplicativo; d) na frase colocada em seu status do aplicativo (visto que alguns inscritos adotavam frases com dizeres de alerta, de dor, tristeza e de sofrimento);e) através da percepção de parâmetros clínicos em saúde mental e de análise de risco (choro inconsolável; discursos de ideação suicida ou auto mutilação; discursos sugestivos de automedicação ou abuso de substâncias como álcool; autonegligência; ansiedade intensa, pânico e impulsividade com risco para si e/ou terceiros; insônia; agressividade, etc.), assim, quando esses sinais eram identificados imediatamente o inscrito era atendido. 
Em seguida, o(s) inscrito(s) recebiam uma ligação telefônica do profissional enfermeiro designado para atendê-lo no dia e horário agendado. $O$ teor das ligações teve durabilidade (entre 15 a 120 minutos) e temáticas definidas e conduzidas pelo próprio inscrito atendido, cabendo, ao enfermeiro responsável pelo atendimento, acolher queixas, relatos, sofrimentos e necessidades apresentadas por aquele(s) inscrito(s), fornecendoIhe(s) escuta, segurança, confidencialidade, profissionalismo e suporte em aspectos de vida, saúde, trabalho e enfrentamento da pandemia COVID-19. Não foram estabelecidos números mínimos e máximos de atendimentos, assim, ao final de cada atendimento era facultado aos inscritos um novo agendamento, conforme sua necessidade (semanal, quinzenal ou mensal).

No referido período, alguns inscritos foram atendidos duas vezes por semana; outros foram atendidos somente por mensagens (aplicativo de comunicação) e houve demais inscritos que desejaram apenas um único atendimento. Ressalta-se que: 1) nenhum inscrito deixou de ser acolhido e orientado (mesmo que sua demanda inicial fosse destinada a outros setores do COREn-MG (como setor financeiro, de denúncias, entre outros); 2) caso o inscrito não se sentisse à vontade com o profissional que $o$ atendeu, Ihe era facultado experienciar $o$ atendimento de outro profissional; 3 ) inscritos com mais de dois atendimentos eram mantidos no mesmo dia semana e horário (respeitando sua rotina profissional) e eram atendidos (se assim desejassem) pelo mesmo profissional); e 4) caso a ligação não fosse atendida no dia e no horário marcado, o profissional responsável pelo atendimento realizava três tentativas de ligação, com intervalos de 30 minutos entre elas, e, se mesmo assim, por algum motivo o inscrito não atendesse, era feito um novo contato e novo agendamento. Salienta-se, que o relato sobre a sobrecarga de trabalho vivida por muitos profissionais foi motivo de impedimento para muitos atendimentos, pois, mesmo com dia e horário agendados, estes não conseguiram receber o suporte, ora por ter sido convocados de última hora para cobrir algum colega de plantão, ora, porque terminavam sua jornada de trabalho muito além do seu horário pré-estabelecido.

A equipe de integrantes da CSEE, denominados como colaboradores, foi composta por 3 enfermeiros e 16 enfermeiras, capacitados para o desenvolvimento das diversas atividades da CSEE, como a formulação de estratégias de divulgação do serviço aos profissionais do estado de Minas Gerais; organização e gestão dos atendimentos; avaliação, discussão de caso e encaminhamentos, quando necessários; manejo do aplicativo de comunicação e agendamentos e avaliação dos atendimentos através da realização de uma pesquisa de satisfação. Além da escuta profissional, ao final do atendimento, o inscrito era convidado a participar de uma pesquisa sobre condições de trabalho, vida e saúde realizada por esta comissão, por meio de aprovação da Comissão Nacional de Ética em Pesquisa (CONEP).

A pesquisa de satisfação foi realizada na semana posterior aos atendimentos, via ligação telefônica (até três tentativas), utilizando-se o Net Promoter Score (NPS) ${ }^{6}$ que mensura a satisfação dos clientes/pacientes em relação a um serviço/ produto. É questionado aos clientes/pacientes uma pontuação (0 a 10 pontos) acerca de sua satisfação, estratificando-os em três categorias: a) promotores ( 9 a 10 pontos): gostaram do atendimento, ficaram satisfeitos e incentivaram outros indivíduos a participarem do suporte; b) neutros (7 a 8 pontos): não ajudaram e não atrapalharam na divulgação do suporte; $\mathrm{c}$ ) detratores ( 0 a 6 pontos): insatisfeitos, não tiveram uma boa relação e chegaram a prejudicar a imagem do suporte. Ao final, o NPS é calculado com a subtração da porcentagem obtida entre os promotores e detratores (\% promotores - \% detratores) e classifica a satisfação em:zona de excelência ( $75 \%$ a $100 \%)$; zona de qualidade (50\% a $74 \%)$; zona de aperfeiçoamento $(49 \%$ a $0 \%)$ e zona crítica $(-100 \%$ a $-1 \%)$.

Para o desenvolvimento do trabalho, os colaboradores vivenciaram capacitações e reuniões mensais, espaços para comunicação sobre o processo de trabalho, discussão e supervisão de casos. Destaca-se que, de forma singular, acordado entre colaboradores e inscritos, houve interlocuções e encaminhamentos para serviços da rede de saúde mental do município de referência do profissional atendido, a fim de fornecer um maior acompanhamento para casos com maior agravamento. Tal fato foi possível dada a inserção anterior dos colaboradores na rede de saúde mental da capital e região metropolitana ou de levantamento realizado com lista pré-distribuída aos colaboradores sobre serviços disponíveis nas regiões do estado, possibilitando conexões entre os atendidos e serviços de referências. Ademais, cabe ressaltar que grande parte dos inscritos atendidos relataram histórico de acompanhamento em saúde mental. Contudo, optaram, adicionalmente aos seus planos terapêuticos, pelo suporte ético-emocional, considerando a identificação e empatia profissional, haja vista que foi um serviço oferecido por enfermeiros e enfermeiras para profissionais de enfermagem, em que poderiam falar também sobre problemas vivenciados no ambiente de trabalho com maior fluidez de comunicação.

Para a avaliação do padrão clínico-emocional apresentado pelo paciente, os colaboradores dispunham de um protocolo de classificação de risco em saúde mental ${ }^{7}$, além de testes internacionais validados no contexto brasileiro, como o AUDIT-C ${ }^{8}$ e o DASS- $21^{9}$, que dialogam acerca do padrão de consumo de álcool e sintomas de depressão, ansiedade e estresse, respectivamente. Para além do uso dos critérios supracitados, o padrão clínico-emocional era avaliado em cada ligação através de relatos verbais, expressão das emoções, assim como pelo tom de voz, visto que tratava de um acolhimento à distância, fato que impossibilitou uma avaliação mais ampla. Vale ressaltar que os atendimentos e o suporte éticoemocional realizado por enfermeiros têm como base legal a lei ำ 7498/86 (que regulamenta a atuação da Enfermagem) ${ }^{10}$; a resolução do COFEN no 599/2018 (que estabelece os parâmetros para atuação ética, segura e humanizada em Saúde Mental) ${ }^{11}$; e a resolução do COFEN no 564/2017 (que delineia e explicita o Código de Ética dos Profissionais de Enfermagem) ${ }^{12}$. Assim, em todos os momentos, os aspectos éticos-legais que permeiam a consulta de enfermagem em Saúde Mental foram respeitados, 
tendo por base as supracitadas normativas. Ademais, este relato de experiência recebeu aprovação pela Comissão Nacional de Ética em Pesquisa sob o parecer n 4.169.027 e CAAE 32019320.4.0000.0008.

\section{RESULTADOS DA EXPERIÊNCIA}

\section{Promoção da saúde mental frente à pandemia de COVID-19: vivências dos inscritos atendidos pela CSEE}

Foram atendidos 241 profissionais de enfermagem pelo suporte, dentre eles, profissionais do nível técnico e mulheres constituíram a maioria dos inscritos que buscaram pelo serviço. Cabe ressaltar que, dado o caráter sigiloso dos atendimentos e de forma a assegurar o anonimato e proteção dos inscritos, foi-Ihes garantido o uso de pseudônimos, assim como a não identificação dos mesmos. Portanto, não foi possível detalhar outros elementos acerca da caracterização dos profissionais atendidos. Destaca-se que o COREn-MG dispõe de uma sede em Belo Horizonte e nove subseções em municípios do estado, além de 19 representantes, em processo de expansão de sua representação de forma presencial, nos 853 municípios mineiros, e possui 219.175 profissionais inscritos, sendo a maior parte destes, técnicos ( $n=133.389 ; 60,9 \%)$; seguidos por enfermeiros
( $n=55.442 ; 25,3 \%)$; auxiliares $(n=19.750 ; 9 \%)$ e atendentes $(n=10.594 ; 4,8 \%)^{13}$.

Durante os atendimentos, foram reveladas e descritas emoções, sentimentos, vivências e problemas oriundos do novo contexto pandêmico. Os problemas relatados foram frequentes durante os atendimentos da CSEE e reforçam a eminência, a urgência e o valor que o suporte ético-emocional teve durante a pandemia para a vida, para o trabalho e para a saúde dos profissionais de enfermagem, pois, para muitos o suporte foi companhia, salvamento, amparo e "válvula de escape". Além disso, os problemas vivenciados e relatados sofreram forte influência da sazonalidade e das especificidades de prevenção e controle da COVID-19 adotadas em cada município mineiro. Foi observado, por exemplo, que a abertura do comércio trazia aos profissionais de saúde sentimentos significativos de ansiedade, medo e insegurança, visto que, tal expansão de circulação representava também um maior risco de infecção, que consequentemente resultava na superlotação dos serviços de saúde e na sobrecarga física e emocional no trabalho (Figura 1).

As temáticas trabalhadas durante os atendimentos seguiram os fundamentos teóricos do campo da saúde mental ${ }^{14}$, utilizandose dos conceitos e prática de abordagem centrada na pessoa e compartilhamento de decisões. Sendo assim, essas incluíram: a) identificação e conduta imediata em situações emergenciais e prioritárias; b) reconhecimento e atenção às subjetividades dos profissionais atendidos; c) criação e estabelecimento de um plano

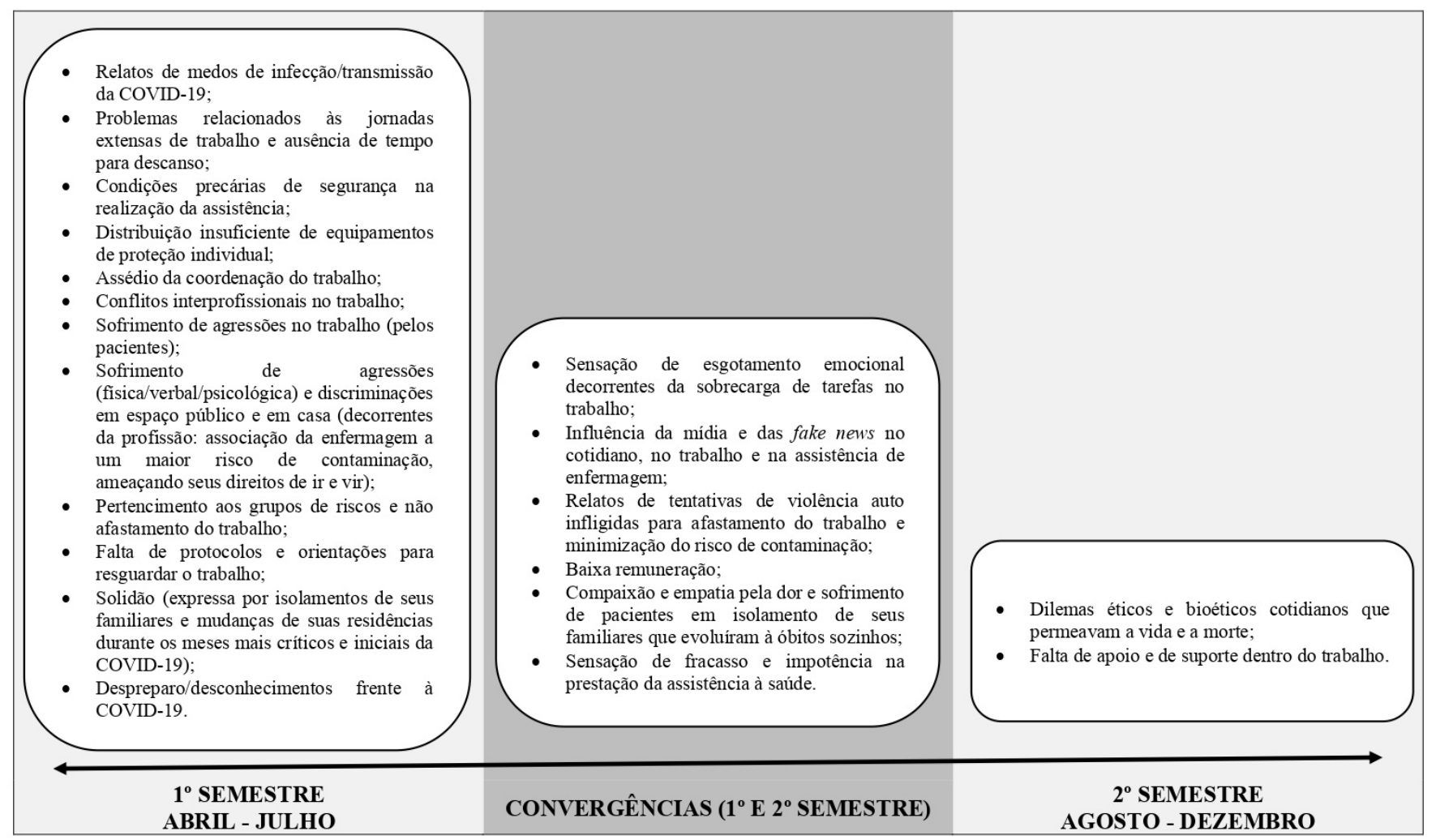

Figura 1. Situações vivenciadas pelos profissionais de enfermagem frente à pandemia de COVID-19. Minas Gerais, Brasil, 2020. 
terapêutico junto ao inscrito com ferramentas de enfrentamento e estratégias de precauções para evitar recidivas acerca de crises de ansiedade e estresse no trabalho; d) orientações para busca de informações, prevenção e controle da COVID-19 no próprio site do COREn-MG; e) orientações e instruções para a utilização adequada e saudável de informações sobre a COVID-19 (essa estratégia fez-se essencial para estabilidade e controle emocional, evitando-se os "gatilhos"); f) sugestão do uso de técnicas de relaxamento ou práticas integrativas e complementares (acupuntura, meditação, reflexologia, reiki etc.) para fins de prevenção, controle, manejo e enfrentamento da ansiedade, dos medos, sintomas psicossomáticos, das preocupações e demais sintomas que emergiram ou se atenuaram durante a pandemia de COVID-19.

A indispensabilidade e a importância do suporte éticoemocional foram retratadas e afirmadas através dos resultados do NPS, em que $84 \%$ das pessoas que responderam à pesquisa de satisfação se declararam satisfeitas e que indicariam o serviço para colegas (promotores); seguidos por 11\% que mantiveram uma neutralidade em relação a satisfação e divulgação do suporte (neutros); e apenas 5\% ficaram insatisfeitos ou não tiveram uma boa relação com o suporte prestado (detratores). Tal resultado coloca o serviço de suporte ético-emocional na zona de excelência, obtendo um escore do NPS de 79\%, e pode ser exemplificado pelos depoimentos abaixo colhidos:

[...] A pessoa que me atendeu foi um anjo que me ligou no momento, que eu tanto precisava. Estava prestes a fazer um teste rápido, fiquei muito insegura, coração disparado pois tenho filhos e fiquei com medo de estar doente. Gostei tanto que solicitei um novo atendimento com ela. Ela me ajudou muito, me resgatou a profissional que sou, elevou minha autoestima. Gostei demais do atendimento dela, me deixou segura, me tirou o medo. Nem sei explicar o quanto foi útil para mim este suporte. Obrigada! [...] (Inscrito 1).

[...] O conselho pela primeira vez disponibilizou um serviço que a classe de Enfermagem precisa, pois nós somos profissionais que temos muitos desequilíbrios pelo desgaste de nossa profissão. Temos muitas dificuldades de enfrentamento, principalmente agora nesta crise, vendo nossos colegas morrendo. Pela primeira vez vi o conselho interagindo conosco além de demandas burocráticas como as de carteira profissional. Nós realmente precisávamos disso e pela primeira vez me senti realmente acolhida! Gostei tanto que agendei novamente [...] (Inscrito 2).

[...] A menina me ajudou bastante, conversamos coisas da minha vida além da pandemia, me senti muito mais confiante! Eu vou falar para você que tem mais de doze anos que estou na área da Enfermagem e nunca vi um programa tão maravilhoso como este! Agradeço imensamente e quando eu precisar de novo enviarei um WhatsApp [aplicativo de comunicação]! Vocês devem divulgar mais nas redes sociais! [...] (Inscrito 3).
[...] Pois é bom a gente conversar com alguém que nos entende, conversar com familiar ou com amigos quando estamos perdidos às vezes é difícil, que não compreende. Quando é um profissional da Enfermagem, que tem a preparação para este tipo de escuta e que faz com profissionalismo, nos sentimos mais seguros. Foi muito bom o atendimento [...] (Inscrito 4).

Deste modo, o suporte ético-emocional desenvolvido pela CSEE configurou-se como uma estratégia de saúde e de intervenção indispensável à promoção, prevenção, segurança e proteção da saúde mental dos profissionais de enfermagem frente ao cotidiano imposto pela COVID-19.

\section{O trabalho em saúde mental de enfermeiros e enfermeiras frente à pandemia de COVID-19: atuação dos colaboradores da CSEE}

Frente ao contexto emergencial, a CSEE trabalhou utilizandose de estratégias de abordagem multifacetadas e de parâmetros para atuação ética, segura e humanizada em saúde mental, o que resultou num fazer concreto baseado na(s) realidade(s) e na(s) subjetividade (s)/necessidade(s) dos profissionais inscritos. Ressalta-se que, para a execução e excelência do trabalho prestado, a seleção de uma equipe qualificada fez-se essencial.

Durante o período de atuação da CSEE, observou-se a construção de vínculos e da confiança entre o profissional enfermeiro (colaborador) e o profissional inscrito atendido. Salienta-se que os atendimentos se apoiaram na escuta qualificada; humanização; exercício da empatia compartilhada e da comunicação não-violenta, com o respeito às singularidades, diversidades e potencialidades dos seres humanos, cumprindo-se o sigilo e o anonimato dos profissionais atendidos, o que acabou por culminar na redução de sentimentos negativos; na percepção aumentada (para identificação de potenciais ameaças e contextos de "gatilhos") na valorização pessoal; no autoconhecimento e no autocuidado. Como particularidades do suporte ético-emocional, observa-se, por exemplo, a não continuidade de um próximo atendimento, visto que, alguns inscritos não desejavam mais o suporte para aquele momento, pois o primeiro atendimento trouxe a percepção de sentir-se bem e acolhido em suas necessidades, obtendose a resolubilidade esperada para sua demanda, sendo assim respeitada a autonomia e desejo deste, mantendo-se o suporte sempre disponível.

Ademais, durante os atendimentos, o profissional enfermeiro (colaborador) utilizava-se da Sistematização da Assistência de Enfermagem e suas ferramentas tais como a anotação de enfermagem para acompanhar a evolução o progresso e as intervenções individuais dos inscritos atendidos, assim como a utilização de escalas e de instrumentos associados à saúde mental (escalas sobre o padrão de consumo de álcool e sintomas de depressão, ansiedade e estresse).

Completando-se, o modelo de atendimento proposto pela CSEE baseava-se: no matriciamento semanal dos casos atendidos; na supervisão e treinamentos da equipe; na elaboração 
de relatórios mensais dos atendimentos, assim como na troca diária de experiências e feedbacks entre os colaboradores, nas reuniões periódicas e em grupo de comunicação por aplicativo.

\section{REFLEXÕES DA EXPERIÊNCIA}

Sobreleva-se que trabalhar com promoção e a proteção da Saúde Mental em tempos de COVID-19 foi um desafio gigantesco, mas, que no final fez-se recompensador.

Salienta-se que o caráter gratuito desse serviço se fez acolhedor, principalmente, devido às repercussões socioeconômicas ocasionadas pelas infecções por coronavírus, e consequentemente pela COVID-19. O fato de a CSEE operacionalizar-se por agendamentos via ligações telefônicas e aplicativo de comunicação e por possuir horários vastos de atendimento (de segunda a sexta-feira das 8 às 21 horas) Ihe conferiu um caráter flexibilizador para procura dos profissionais de Enfermagem.

Como fortaleza e caráter inovador deste relato, destaca-se que a CSEE ofertou uma classificação de risco à distância e permitiu a identificação de riscos para a saúde mental através das falas, da escuta, do silêncio e dos relatos por mensagem, por áudio ou por ligação telefônica. O aplicativo de comunicação se fez muito útil neste percurso, ao se firmar como instrumento facilitador da CSEE, principalmente por permitir comunicações e agendamentos profícuos, que foram capazes de evitar falhas, imprecisões e afligimentos entre os inscritos e toda a equipe da CSEE. Apesar da grande extensão geográfica de Minas Gerais e do caráter institucional (COREn-MG) do suporte, ressalta-se que a implementação de atendimentos de telessaúde, como os operacionalizados pela CSEE, tem potencial para serem desenvolvidos em diversos contextos sociais, desde que tenha profissionais qualificados para seu manejo. Essas ações podem ser aplicadas e oferecidas às diversas categorias profissionais (não somente aos profissionais de saúde) e a população em geral, pois, tal intervenção caracteriza-se por técnicas de acolhimento e comunicação empáticas, não violentas, não farmacológicas e de baixo custo e apresentam potencial para uso individual e coletivo, fazendo-se indispensáveis frente aos danos emocionais e enfrentamento da pandemia de COVID-19.

\section{CONSIDERAÇÕES FINAIS E IMPLICAÇÕES PARA A PRÁTICA}

Este relato reforça a premissa: "onde há vida, há Enfermagem!" Pois, independente do contexto social vivido, do espaço (presencial ou online), do público a ser cuidado e da atividade do cuidado a ser executada (consulta, gerência, assistência, educação ou pesquisa), a Enfermagem, mostra-se extraordinariamente multisciente e habilitada.

Dessarte, o suporte explicitado neste relato fez-se inovador para área da saúde e da enfermagem ao constituir-se como uma estratégia multidisciplinar promotora, aconselhadora e facilitadora do cuidado em tempos de COVID-19. Encoraja-se a manutenção desta estratégia após a pandemia.
Ademais, a divulgação dessa experiência nos dias atuais, fomenta novas reflexões, discussões e informações capazes de direcionar e beneficiar ações de promoção da saúde mental entre trabalhadores de saúde.

Verifica-se a ausência de dados e do histórico clínico dos inscritos atendidos, além do fato de que a observação presencial da linguagem e das expressões corporais não foram possíveis, devido ao contexto de isolamento do inscrito e do profissional enfermeiro(a) (colaborador). Contudo, tais implicações não se configuraram como limitações significativas, uma vez que, mesmo que a distância o potencial terapêutico foi atingido, devido sobretudo, ao envolvimento interpessoal, desenvolvido através da escuta e da permanência semanal, quinzenal ou mensal do profissional enfermeiro(a) na vida dos inscritos.

\section{CONTRIBUIÇÃO DOS AUTORES}

Desenho do relato de experiência. Gabriela Gonçalves Amaral. Lívia Silveira Silva. Jarbas Vieira de Oliveira. Noelle Melo Machado. Juliana Silveira Teixeira. Hozana Reis Passos.

Levantamento de informações. Lívia Silveira Silva. Jarbas Vieira de Oliveira. Noelle Melo Machado. Juliana Silveira Teixeira. Gabriela Gonçalves Amaral. Hozana Reis Passos.

Análise de dados. Lívia Silveira Silva. Jarbas Vieira de Oliveira. Noelle Melo Machado. Juliana Silveira Teixeira. Gabriela Gonçalves Amaral. Hozana Reis Passos.

Interpretação dos resultados. Lívia Silveira Silva. Jarbas Vieira de Oliveira. Noelle Melo Machado. Juliana Silveira Teixeira. Gabriela Gonçalves Amaral. Hozana Reis Passos.

Redação e revisão crítica do manuscrito. Gabriela Gonçalves Amaral. Lívia Silveira Silva. Jarbas Vieira de Oliveira. Noelle Melo Machado. Juliana Silveira Teixeira. Hozana Reis Passos.

Aprovação da versão final do artigo. Gabriela Gonçalves Amaral. Lívia Silveira Silva. Jarbas Vieira de Oliveira. Noelle Melo Machado. Juliana Silveira Teixeira. Hozana Reis Passos.

Responsabilidade por todos os aspectos do conteúdo e a integridade do artigo publicado.

Gabriela Gonçalves Amaral. Lívia Silveira Silva. Jarbas Vieira de Oliveira. Noelle Melo Machado. Juliana Silveira Teixeira. Hozana Reis Passos.

\section{EDITOR ASSOCIADO}

\author{
Antonio José de Almeida Filho (1)
}

\section{EDITOR CIENTÍFICO}

\author{
Ivone Evangelista Cabral ๑)
}

\section{REFERÊNCIAS}

1. Viegas SMF. Lâmpada que não se apaga: enfermagem em prol do reconhecimento social e valorização de seus profissionais. HU Rev. 2021;46:1-2. http://dx.doi.org/10.34019/1982-8047.2020.v46.32774.

2. Gomes RLE, Faria LL, Holzmann HA, Fujiwara NKF, Ando SM, Sawamura MVY et al. Fighting the invisible enemy: providing support and structure to radiology resident during the COVID-19 pandemic. Radiol Bras. 
2020;53(6):397-400. http://dx.doi.org/10.1590/0100-3984.2020.0067. PMid:33304007.

3. Portaria no 166 , de 30 de março de 2020 (BR). Cria e constitui Comissão de Suporte Ético-Emocional - CSEE, no âmbito do Conselho Regional de Enfermagem de Minas Gerais com o objetivo de elaborar recomendações, orientações e suporte ético e emocional em Saúde Mental relacionados aos profissionais de enfermagem considerando a Pandemia de COVID-19, e dá outras providências. Diário Oficial da União [periódico na internet], Brasília (DF), 31 mar. 2020 [citado 14 mar 2021]. Disponível em: https://sig.corenmg.gov.br/sistemas/file/ doc/legislacoes/docs/doc_legis_2897.pdf

4. Humerez DC, Ohl RIB, Silva MCN. Mental health of brazilian nursing professionals in the context of the covid-19 pandemic: action of the Nursing Federal Council. Cogitare Enferm. 2020;25:e74115. http:// dx.doi.org/10.5380/ce.v25i0.74115.

5. Daltro MR, Faria AA. Relato de experiência: Uma narrativa científica na pós-modernidade. Estud Pesqui Psicol. 2019;19(1):223-37. http:// dx.doi.org/10.12957/epp.2019.43015.

6. Reichheld FF. The one number you need to grow. Harvard Business Review. 2003 dez [citado 11 mar 2021]. Disponível em: https://hbr. org/2003/12/the-one-number-you-need-to-grow

7. Secretaria de Saúde (ES). Protocolo estadual de classificação de risco em saúde mental [Internet]. Vitória: SES-ES; 2018 [citado 31 ju 2021]. Disponível em: https://saude.es.gov.br/Media/sesa/Consulta\%20 P\%C3\%BAblica/PROTOCOLO\%20CLASSIFICACAO\%20DE\%20 RISCO\%20EM\%20SAUDE\%20MENTAL_CONSULTA.pdf

8. Babor TF, Higgins-Biddle J, Saunders JB, Monteiro MG. AUDIT: teste para identificação de problemas relacionados ao uso de álcool: roteiro para uso na atenção primária. Ribeirão Preto: Programa de Ações
Integradas para Prevenção e Atenção ao Uso de Álcool e Drogas na Comunidade; 2003.

9. Vignola RCB, Tucci AM. Adaptation and validation of the depression, anxiety and stress scale (DASS) to Brazilian Portuguese. J Affect Disord. 2014;155:104-9. http://dx.doi.org/10.1016/j.jad.2013.10.031. PMid:24238871.

10. Lei n. $7498 / 86$ de 25 de junho de 1986 (BR). Dispõe sobre a regulamentação do exercício da Enfermagem e dá outras providências. Diário Oficial da União [periódico na internet], Brasília (DF), 26 jun. 1986 [citado 14 mar 2021]. Disponível em: http://www.cofen.gov.br/lei-n-749886-de25-de-junho-de-1986_4161.html

11. Resolução n. 599/2018 (BR). Dispõe sobre o estabelecimento de parâmetros mínimos para assistência segura e humanizada na Enfermagem em Saúde Mental. Diário Oficial da União [periódico na internet], Brasília (DF), 21 dez. 2018 [citado 14 mar 2021]. Disponível em: http://www.cofen.gov.br/resolucao-cofenno-599-2018_67820.htm

12. Resolução n. 564/2017 (BR). Aprova o Código de ética dos profissionais de Enfermagem. Diário Oficial da União [periódico na internet], Brasília (DF), 6 dez. 2017 [citado 14 mar 2021]. Disponível em: http://www. cofen.gov.br/resolucao-cofen-no-5642017_59145.html

13. Conselho Regional de Enfermagem de Minas Gerais. Quem somos [Internet]. Belo Horizonte: COREN-MG; 2020 [citado 24 jul 2021]. Disponível em: https://www.corenmg.gov.br/quem-somos/

14. Ministério da Saúde (BR), Secretaria de Atenção à Saúde, Departamento de Atenção Básica. Saúde mental [Internet]. Brasília: MS; 2013. (Cadernos de Atenção Básica; no. 34) [citado 13 mar 2021]. Disponível em: http:// bvsms.saude.gov.br/bvs/publicacoes/cadernos_atencao_basica_34_ saude_mental.pdf 